\title{
Mean square exponential and non-exponential asymptotic stability of impulsive stochastic Volterra equations
}

\author{
Dianli Zhao ${ }^{1,2^{*}}$ and Dong Han ${ }^{1}$
}

\author{
* Correspondence: \\ tc_zhaodianli@139.com \\ 'Department of mathematics, \\ Shanghai Jiaotong University, \\ Shanghai, 200240, China \\ Full list of author information is \\ available at the end of the article
}

\begin{abstract}
In this article, some inequalities on convolution equations are presented firstly. The mean square stability of the zero solution of the impulsive stochastic Volterra equation is studied by using obtained inequalities on Liapunov function, including mean square exponential and non-exponential asymptotic stability. Several sufficient conditions for the mean square stability are presented. Results in this article indicate that not only the impulse intensity but also the time of impulse can influence the stability of the systems. At last, an example is given to show application of some obtained results.

Mathematics classification Primary(2000): 60H10, 60F15, 60J70, 34F05.
\end{abstract}

Keywords: Stochastic Volterra equations, Impulse, Liapunov function, Asymptotic stability

\section{Introduction}

Study on the stability of stochastic differential equations has gained lots of attention over the last years. The results and methods have been improved from time to time. Very recently, Taniguchi [1] studied the exponential stability for stochastic delay partial differential equations by use of the energy method which overcomes the difficulty of constructing the Liapunov functional on delay differential equations. Wan and Duan [2] extended the result of Taniguchi [1] to be applied to more general stochastic partial differential equations with memory. Another important method is about the fixedpoint theory. It was first used to consider the exponential stability for stochastic partial differential equations with delays by Luo [3], where the conditions do not require the monotone decreasing behavior of the delays. This method also employed in Sakthivel and Luo $[4,5]$ to study the asymptotic stability of the nonlinear impulsive stochastic differential equations and the impulsive stochastic partial differential equations with infinite delays.

On considering Volterra equations, there is a significant literature devoted to the asymptotic stability of the zero solutions of Volterra integro-differential equations. In the known literature, the properties of linear scalar Voterra equation play an important role. The equation is

\section{SpringerOpen ${ }^{\circ}$}

(C) 2011 Zhao and Han; licensee Springer. This is an Open Access article distributed under the terms of the Creative Commons Attribution License (http://creativecommons.org/licenses/by/2.0), which permits unrestricted use, distribution, and reproduction in any medium, provided the original work is properly cited. 


$$
x^{\prime}(t)=-a x(t)+\int_{0}^{t} k(t-s) x(s) \mathrm{d} s
$$

where the kernel $k(t)$ is continuous, integrable and of a single sign. Brauer [6] showed that the solution could not be stable if $\int_{0}^{\infty} k(s) \mathrm{d} s>a$, Burton and Mahfoud [7] proved the zero solution is asymptotically stable if $\int_{0}^{\infty} k(s) \mathrm{d} s<a$, Kordonis and Philos [8] discussed the stability of the solution under condition $\int_{0}^{\infty} k(s) \mathrm{d} s=a$. Therefore, a necessary condition for $\lim _{t \rightarrow \infty} x(t)=0$ for all solutions is that $\int_{0}^{\infty} k(s) \mathrm{d} s \leq a$. About exponential asymptotic stability, Murakami [9] showed that the uniform asymptotic stability and the exponential asymptotic stability of the zero solution of this equation are equivalent if and only if $\int_{0}^{\infty} k(s) \exp (\gamma s) \mathrm{d} s<\infty$ for some $\gamma>0$. Hence if it fails to hold, a uniformly asymptotically stable solution cannot be exponentially asymptotically stable. Some deeper related work on deterministic equations by Appleby can be found in [10-12], including the so-called "non-exponential decay rate" and "subexponential solution". Mao [13] investigated the mean square stability of the generalized equation

$$
\mathrm{d} X(t)=\left(f(t, X(t))+g\left(t, \int_{0}^{t} G(t, s) X(s) \mathrm{d} s\right)\right) \mathrm{d} t+h\left(t, \int_{0}^{t} H(t, s) X(s) \mathrm{d} s\right) \mathrm{d} B(t)
$$

On some special stochastic volterra equations without impulse, we highlight here the contribution of Appely [14-19]. However real-world systems can be modeled to include random effects, including stochastic perturbations and impulses. It is natural to ask how the presence of such random effects can influence the stability of the systems. Based on the generalized equation [13], in this article, we consider the effect of the impulse intensity and the impulse time on the mean square exponential and non-exponential asymptotic stability of impulsive stochastic Volterra equation

$$
\begin{cases}\mathrm{d} x(t)=f\left(t, x(t), \int_{\tau_{i}}^{t} F(t-s) x(s) \mathrm{d} s\right) \mathrm{d} t+g\left(t, x(t), \int_{\tau_{i}}^{t} G(t-s) x(s) \mathrm{d} s\right) \mathrm{d} B(t), & t \in\left(\tau_{\mathrm{i}}, \tau_{\mathrm{i}+1}\right) \\ \Delta x\left(\tau_{i}\right)=I_{i}\left(\xi_{i}, x\left(\tau_{i}^{-}\right)\right) & t=\tau_{\mathrm{i}} \\ x\left(t_{0}\right)=x_{0} & \end{cases}
$$

for all $i \in N=\{0,1,2, \ldots .$.$\} by using Liapunov function, which show that both the$ presence of impulses and the time of the presence can influence the stability of the systems. By choosing the impulse intensity and the impulse time, We find that $\int_{0}^{\infty} k(s) \exp (\gamma s) \mathrm{d} s<\infty$ is not necessary condition for the exponential asymptotic stability.

The article is organized as follows: some preliminary notations and useful lemmas are given in Sect. 2. Then, sufficient conditions of the mean square exponential asymptotic stability are shown in the first part of Sect. 3, and the second part mainly deals with the mean square non-exponential asymptotic stability of the solution. Finally, an example is given.

\section{Preliminary notes}

Let $\left\{\tau_{i}, i=1,2, \ldots\right\}$ be a series of numbers such that $t_{0}=\tau_{0}<\cdots<\tau_{\mathrm{k}}<\tau_{k+1}<\cdots$ and . We denote $R^{+}=[0,+1)$. Consider the impulsive stochastic Volterra equations 


$$
\begin{cases}\mathrm{d} x(t)=f\left(t, x(t), \int_{\tau_{i}}^{t} F(t-s) x(s) \mathrm{d} s\right) \mathrm{d} t+g\left(t, x(t), \int_{\tau_{i}}^{t} G(t-s) x(s) \mathrm{d} s\right) \mathrm{d} B(t), & t \in D_{\mathrm{i}} \\ \Delta x\left(\tau_{i}\right)=I_{i}\left(\xi_{i}, x\left(\tau_{i}^{-}\right)\right), & t=\tau_{i} \\ x\left(t_{0}\right)=x_{0} & \end{cases}
$$

where $D_{i}=\left(\tau_{\mathrm{I}}, \tau_{\mathrm{i}+1}\right)$ for all $i \in N . f(t, x, y): R^{+} \times R^{n} \times R^{n} \rightarrow R^{n}, g(t, x, y): R^{+} \times R^{n} \times$ $R^{n} \rightarrow R^{n} . \xi_{i}=\tau_{i}-\tau_{i-1}, \Delta x\left(\tau_{i}\right)=x\left(\tau_{i}\right)-x\left(\tau_{i}^{-}\right), x\left(\tau_{i}^{-}\right)=\lim _{t \rightarrow \tau_{i}-0} x(t)$ with respect to probability distribution for all $i=1,2, \ldots . I_{i}(t, x): R^{+} \times R^{n} \rightarrow R^{n} . F(t)$ and $G(t)$ are both continuous and integrable matrix-valued functions on $\mathrm{R}^{+} . \mathrm{B}(\mathrm{t})$ is standard $n$-dimensional Brownian motion on a complete filtered probability space $\Omega, F,\left(F^{B}(t)\right)_{\mathrm{t} \geq 0}, P$ ), where the filtration is defined as $F^{B}(t)=\sigma(B(s): 0 \leq s \leq t)$. Almost sure events are Palmost sure in this article denoted by "a.s.". Suppose $f(t, 0, y)=0, g(t, 0, y)=0$ and $I_{i}(t, 0)=0$ for $t>t_{0}$, then $x(t) \equiv 0$ is the solution of (1), which is called zero solution of (1). In this article, we always assume there exists a unique stochastic process satisfying (1), and assume all solutions of (1) are continuous on the left and limitable on the right. We further recall the various standard notions of stability of the zero solution required.

Definition 2.1. The zero solution of (1) is said to be

(i) mean square asymptotically stable, if for any $\varepsilon>0$, there exist constants $\delta>0$ and $T=T\left(t_{0}, \varepsilon\right)>0$ such that $E\left(\|x(t)\|^{2}\right)<\varepsilon$ for all $t>t_{0}+T$ when $E\left(\left\|x_{0}\right\|^{2}\right)<\delta$.

(ii) mean square exponentially asymptotically stable, if for any $t_{0} \in R^{+}$there exist $\lambda>$ $0, T>0$ and $C=C\left(x_{0}, t_{0}\right)>0$ such that $E\left(\|x(t)\|^{2}\right)<C \exp (-\lambda t)$ for $t>T$.

(iii) mean square non-exponentially asymptotically stable, if $\lim _{t \rightarrow \infty} E\left(\|x(t)\|^{2}\right)=0$ and $\lim _{t \rightarrow \infty} \frac{\log \left(E\left(\|x(t)\|^{2}\right)\right)}{t}=$ ohold.

Suppose that $a \vee b=\max \{a, b\}, E(x)$ is the expectation of $x$ and $\|x(t)\|$ is some norm in the sequel. Let $C^{1}[0, \infty)$ be the family of all continuous functions on $[0, \infty)$ which are once continuously differentiable and $C^{1,2}\left(R^{+} \times R^{\mathrm{n}}, R^{+}\right)$denote the family of all nonnegative functions from $R^{+} \times R^{n}$ to $R^{n}$ which are once continuously differentiable in $t$ and twice in $x$. For each $V \in C^{1,2}\left(R^{+} \times R^{n}, R^{+}\right)$, we denote $V(t)=E(V(t, x$ $(t))), V\left(t^{-}\right)=E\left(V\left(t, x\left(t^{-}\right)\right)\right)$and

$$
\begin{aligned}
L V(t, x(t)) & =V_{t}(t, x(t))+V_{x}(t, x(t)) f\left(t, x(t), \int_{\tau_{i}}^{t} F(t-s) x(s) \mathrm{d} s\right) \\
& +\frac{1}{2} \operatorname{trace}\left(g^{T}\left(t, x(t), \int_{\tau_{i}}^{t} G(t-s) x(s) \mathrm{d} s\right) V_{x x}(t, x(t)) g\left(t, x(t), \int_{\tau_{i}}^{t} G(t-s) x(s) \mathrm{d} s\right)\right)
\end{aligned}
$$

where

$$
\begin{gathered}
V_{t}(t, x(t))=\frac{\partial V(t, x(t))}{\partial t} \\
\left.\frac{V(t, x(t))}{\partial x_{i} \partial x_{j}}\right)_{n \times n}
\end{gathered}
$$

and

$V\left(t, x(t)_{x x}=\left(\frac{\partial^{2} V(t, x(t))}{\partial x_{i} \partial x_{j}}\right)_{n \times n}\right.$.

Before going to the main results, let's consider some lemmas about linear Volterra equation without impulses.

Lemma 2.2. Suppose $k(t)>0$ is a function on $R^{+}$. $a>0$ is constant. Let $z(t)$ satisfy

$$
\left\{\begin{array}{l}
z^{\prime}(t)=-a z(t)+\int_{0}^{t} k(t-s) z(s) \mathrm{d} s \\
x(0)=1
\end{array}\right.
$$


Then $z(t)>0$ and $\frac{z(s)}{z(t)} \leq \exp (a(t-s))$ for $t \geq s \geq 0$. Moreover, $\int_{0}^{\infty} k(s) \mathrm{d} s \leq$ aimplies that $z(t) \leq 1$.

Proof. Firstly we claim that $z(t)>0$ for all $t \in[0,+\infty)$, if not, there exists $t>0$ such that $\bar{t}=\inf \{t \geq 0: z(t) \leq 0\}$. Then we have $z(t)>0$ for all $t \in[0, \bar{t})$. Since $0=z(\bar{t})<z(0)$, we get that $z(\bar{t}) \exp (a \bar{t})=0<1=z(0) \exp (a 0)$, then there is $t_{1} \in[0, \bar{t})$ satisfying

$$
\left.(z(t) \exp (a t))^{\prime}\right|_{t=t_{1}}<0
$$

From (2),

$$
\left.(z(t) \exp (a t))^{\prime}\right|_{t=t_{1}}=\exp \left(a t_{1}\right) \int_{0}^{t_{1}} k\left(t_{1}-s\right) z(s) \mathrm{d} s \geq 0
$$

holds, which contradicts with (3). So we get that $z(t)>0$ for all $t \in[0,+\infty)$. Again from (2), we get

$$
(z(t) \exp (a t))^{\prime}=\exp (a t) \int_{0}^{t} k(t-s) z(s) \mathrm{d} s .
$$

By integrating on both sides, we get $\frac{z(s)}{z(t)} \leq \exp (a(t-s))$ for $t \geq s \geq 0$.

If $\int_{0}^{\infty} k(s) \mathrm{d} s \leq a$, by integrating on (2) we get

$$
\begin{aligned}
z(t) & =1-a \int_{0}^{t} z(u) \mathrm{d} u+\int_{0}^{t} \int_{0}^{u} k(u-v) z(v) \mathrm{d} v \mathrm{~d} u \\
& =1-a \int_{0}^{t} z(u)\left(a-\int_{0}^{t-u} k(v) \mathrm{d} v\right) \mathrm{d} u \\
& \leq 1
\end{aligned}
$$

The proof is complete.

Lemma 2.3. [[20], Corollary 3.3] Under conditions in Lemma 2.2. Let $z(t)$ be solution of (2). Suppose

$$
\int_{0}^{\infty} k(s) \mathrm{d} s \leq a .
$$

Then $z(t)$ is nonincreasing on $[0,+\infty)$.

Lemma 2.4. Suppose $k(t)>0$ is a function on $R^{+}$. a $>0$ is constant. $h(t) \geq 0$ is a function on $R^{+}$. Let $y(t)$ satisfy

$$
\gamma^{\prime}(t) \leq-a y(t)+\int_{\tau_{i}}^{t} k(t-s) y(s) \mathrm{d} s+h(t)
$$


for all $t \in\left(\tau_{I}, \tau_{i+1}\right)$ where $i \in N$. Then

$$
y(t) \leq \frac{\gamma\left(\tau_{i}\right)}{z\left(\tau_{i}\right)} z(t)+\int_{\tau_{i}}^{t} z(t-s) h(s) \mathrm{d} s
$$

is true for all $t \in\left(\tau_{I}, \tau_{i+1}\right)$.

Proof. If $\tau_{i}=0$,

$$
\gamma(t)=\gamma(0) z(t)+\int_{0}^{t} z(t-s)(h(s)+g(s)) \mathrm{d} s \leq \gamma(0) z(t)+\int_{0}^{t} z(t-s)(h(s)) \mathrm{d} s
$$

holds for

$$
g(t)=\gamma^{\prime}(t)-\left(-a \gamma(t)+\int_{0}^{t} k(t-s) y(s) \mathrm{d} s+h(t)\right) \leq 0 .
$$

If $\tau_{i}>0$, we have $\int_{0}^{\tau_{i}} k(t-s) z(s) \mathrm{d} s>0$. By supposing $p(t)=\frac{\gamma\left(\tau_{i}\right)}{z\left(\tau_{i}\right)} z(t)+\int_{\tau_{i}}^{t} z(t-s) h(s) \mathrm{d} s$ for all $t \in\left[\tau_{1}, \tau_{i+1}\right)$, we get

$$
p^{\prime}(t)=-a p(t)+\int_{\tau_{i}}^{t} k(t-s) p(s) \mathrm{d} s+\int_{0}^{\tau_{i}} k(t-s) p(s) \mathrm{d} s+h(t)
$$

where $z(t)$ is solution of (2). Now we prove that $y(t) \leq p(t)$ for all $t \in\left[\tau_{i}, \tau_{i+1}\right)$. If it is not true, then there exist $t_{1} \in\left(\tau_{1}, \tau_{i+1}\right)$ such that $y\left(t_{1}\right)>p\left(t_{1}\right)$. Denote $\bar{t}=\inf \left\{t \geq \tau_{i}: \gamma(t)>p(t)\right\}$, then $\gamma(\bar{t})=p(\bar{t})$ and

$$
\gamma(t) \leq p(t)
$$

for all $t \in\left[\tau_{i}, \bar{t}\right)$. Therefore $\left.(\gamma(t)-p(t))^{\prime}\right|_{t=t_{0}^{+}} \geq 0$ holds, which implies

$$
\left.(\gamma(t) \exp (a t))^{\prime}\right|_{t=t_{0}^{+}} \geq\left.(p(t) \exp (a t))^{\prime}\right|_{t=t_{0}^{+}}
$$

From (4), we have

$$
(y(t) \exp (a t))^{\prime} \leq \exp (a t) \int_{\tau_{i}}^{t} k(t-s) y(s) \mathrm{d} s+\exp (a t) h(t)
$$

for all $t \in\left[\tau_{i}, \bar{t}\right)$. From (5), we get

$$
\begin{aligned}
(p(t) \exp (a t))^{\prime} & =\exp (a t) \int_{\tau_{i}}^{t} k(t-s) p(s) \mathrm{d} s+\exp (a t) \int_{0}^{\tau_{i}} k(t-s) p(s) \mathrm{d} s+\exp (a t) h(t) \\
& >\exp (a t) \int_{\tau_{i}}^{t} k(t-s) p(s) \mathrm{d} s+\exp (a t) h(t)
\end{aligned}
$$

for all $t \in\left[\tau_{i}, \bar{t}\right)$. By combining (6)(8) and (9), we obtain $\left.(\gamma(t) \exp (a t))^{\prime}\right|_{t=t_{0}^{+}}<\left.(p(t) \exp (a t))^{\prime}\right|_{t=t_{0}^{+}}$which contradicts with (7). From above all, we arrive at the desired result. 


\section{Main results}

In this section, we consider the nonlinear volterra equation with impulsive effect and denote the solution of (1) by $x(t)$. Several sufficient conditions of mean square stability are presented by comparison method with Liapunov function, which include mean square exponential asymptotic stability and mean square non-exponential asymptotic stability.

\subsection{Mean square exponential asymptotic stability}

Theorem 3.1. If there exist positive numbers $c_{1}, c_{2}$ and $V \in C^{1,2}\left(R^{+} \times R^{n}, R^{+}\right)$satisfying

(i) $c_{1}\|x\|^{p} \leq V(t, x) \leq c_{2}\|x\|^{p}$;

(ii) there exist two continuous and integrable functions $k, h: R^{+} \rightarrow R^{+}$and constant a $>0$ such that

$$
L V(t, x(t)) \leq-a V(t, x(t))+\int_{\tau_{i-1}}^{t} k(t-s) V(s, x(s)) \mathrm{d} s+h(t), t \in\left(\tau_{i-1}, \tau_{i}\right)
$$

for any $i=1,2, \ldots$;

(iii) there exist constants $\omega_{i}$ such that for any $i=1,2, \ldots$, we have

$$
E V\left(\tau_{i}, x\left(\tau_{i}\right)\right) \leq \omega_{i} E V\left(\tau_{i}, x\left(\tau_{i}^{-}\right)\right) ;
$$

(iv) $\prod_{j=1}^{+\infty}\left(1 \vee \omega_{j}\right)<+\infty$ and $\int_{0}^{+\infty} h(s) \exp (a s) \mathrm{d} s<+\infty$;

(v) there exists $\gamma>0$ such that $\int_{0}^{+\infty} k(s) \exp (\gamma s) \mathrm{d} s<+\infty$ and $\int_{0}^{+\infty} k(s) \mathrm{d} s<a$.

Then zero solution of (1) is mean square exponentially asymptotically stable.

Proof. From (ii), we have

$$
D^{+} V(t) \leq-a V(t)+\int_{\tau_{i}}^{t} k(t-s) V(s) \mathrm{d} s+h(t)
$$

where $D^{+}$denotes the right Dini derivative. By Lemma 2.4,

$$
V(t) \leq V\left(t_{0}\right) \frac{z(t)}{z\left(\tau_{i}\right)}+\int_{\tau_{i}}^{t} z(t-s) h(s) \mathrm{d} s
$$

for all $t \in\left[\tau_{i-1}, \tau_{i}\right)$. Now let's prove that

$$
V(t) \leq V\left(t_{0}\right) \frac{z(t)}{z\left(t_{0}\right)} \prod_{l=1}^{i-1} \omega_{l}+\int_{\tau_{i-1}}^{t} z(t-s) h(s) \mathrm{d} s+\sum_{l=1}^{i-1} \frac{z(t)}{z\left(\tau_{l}\right)} \prod_{j=i}^{i-1} \omega_{j} \int_{\tau_{l-1}}^{\tau_{l}} z\left(\tau_{l}-s\right) h(s) \mathrm{d} s
$$

holds for all $t \in\left[\tau_{\mathrm{i}-1}, \tau_{i}\right)$ by mathematical induction for $i=1,2, \ldots$ We stipulate $\prod_{j=1}^{i-1}(\cdot)=1$ and $\sum_{j=1}^{i}(\cdot)=0$ as $i=1$ here and in the sequel. (11) is true for $\mathrm{i}=1$ immediately from (10). Assume that (11) holds for any $i \geq 1$, then for $t=\tau_{\mathrm{i}}$ we get 


$$
\begin{aligned}
V\left(\tau_{i}\right) & \leq V\left(t_{0}\right) \frac{z\left(\tau_{i}\right)}{z\left(t_{0}\right)} \prod_{l=1}^{i} \omega_{l}+\omega_{i} \int_{\tau_{i-1}}^{t} z(t-s) h(s) \mathrm{d} s+\sum_{l=1}^{i-1} \frac{z\left(\tau_{i}\right)}{z\left(\tau_{l}\right)} \prod_{j=i}^{i} \omega_{j} \int_{\tau_{l-1}}^{\tau_{l}} z\left(\tau_{l}-s\right) h(s) \mathrm{d} s \\
& =V\left(t_{0}\right) \frac{z\left(\tau_{i}\right)}{z\left(t_{0}\right)} \prod_{l=1}^{i} \omega_{l}+\sum_{l=1}^{i} \frac{z\left(\tau_{i}\right)}{z\left(\tau_{l}\right)} \prod_{j=i}^{i} \omega_{j} \int_{\tau_{l-1}}^{\tau_{l}} z\left(\tau_{l}-s\right) h(s) \mathrm{d} s
\end{aligned}
$$

From assumption (iii) Then by use of (10) for all $t \in\left[\tau_{i}, \tau_{i+1}\right)$ we get

$$
V(t) \leq V\left(t_{0}\right) \frac{z(t)}{z\left(t_{0}\right)} \prod_{l=1}^{i} \omega_{l}+\int_{\tau_{i}}^{t} z(t-s) h(s) \mathrm{d} s+\sum_{l=1}^{i} \frac{z(t)}{z\left(\tau_{l}\right)} \prod_{j=i}^{i} \omega_{j} \int_{\tau_{l-1}}^{\tau_{l}} z\left(\tau_{l}-s\right) h(s) \mathrm{d} s .
$$

Thus by mathematical induction (11) is true for $i=1,2, \ldots$.

By Lemma 2.2, it follows (11) that

$$
V(t) \leq z(t)\left(\frac{V\left(t_{0}\right)}{z\left(t_{0}\right)} \prod_{l=1}^{\infty}\left(1 \vee \omega_{l}\right)+\int_{t_{0}}^{\infty} h(s) \exp (a s) \mathrm{d} s\right) .
$$

Then the mean square exponential asymptotic stability of (1) inherits from that of solutions of (2) under assumptions (iv) and (v). The proof is complete.

Corollary 3.2. If there exist positive numbers $c_{1}, c_{2}$ and $V \in C^{1,2}\left(R^{+} \times R^{n}, R^{+}\right)$satisfying (i)-(iii) and

(v) in Theorem 3.1 and

(H1) $\int_{0}^{\infty} h(s) \exp (a s) \mathrm{d} s<\infty$;

(H2) there exists $0<\rho<1$ such that $\max _{j \in N \backslash\{0\}}\left\{\omega_{j}\right\} \leq \rho$.

Then zero solution of (1) is mean square exponentially asymptotically stable.

Proof. Since $\max _{j \in N \backslash\{0\}}\left\{\omega_{j}\right\} \leq \rho$ implies $\prod_{j=1}^{+\infty}\left(1 \vee \omega_{j}\right)<+\infty$, the result is proved by Theorem 3.1.

Theorem 3.3. If there exist positive numbers $c_{1}, c_{2}$ and $V \in C^{1,2}\left(R^{+} \times R^{n}, R^{+}\right)$ satisfying

(i) $c_{1}\|x\|^{p} \leq V(t, x) \leq c_{2}\|x\|^{p}$;

(ii) there exist continuous and integrable function $k: R^{+} \rightarrow R^{+}$and positive constant a such that for any $i=1,2, \ldots$

$$
L V(t, x(t)) \leq-a V(t, x(t))+\int_{\tau_{i-1}}^{t} k(t-s) V(s, x(s)) \mathrm{d} s, t \in\left(\tau_{i-1}, \tau_{i}\right)
$$

holds when $E\left\|x\left(\tau_{i-1}\right)\right\|^{2}<\theta$ for some constant $\theta>0$;

(iii) there exist positive constants $\omega_{i}$ such that for any $i=1,2, \ldots$, we have

$$
E V\left(\tau_{i}, x\left(\tau_{i}\right)\right) \leq \omega_{i} E V\left(\tau_{i}, x\left(\tau_{i}^{-}\right)\right) ;
$$

(iv) there exists $0<\rho<1$ such that $\max _{j \in N \backslash\{0\}}\left\{\omega_{j}\right\} \leq \rho_{\text {; }}$

(v) $\int_{0}^{\infty} k(s) \mathrm{d} s \leq a$;

(vi) $\tau_{\mathrm{i}} \leq t_{0}+i$ for all $i \in N$.

Then zero solution of (1) is mean square exponentially asymptotically stable. 
Proof. Assumption (ii) implies

$$
D^{+} V(t) \leq-a V(t)+\int_{\tau_{i}}^{t} k(t-s) V(s) \mathrm{d} s .
$$

From Lemma 2.4, let $h(t)=0$, for all $t \in\left[\tau_{i-1}, \tau_{i}\right)$ we get

$$
V(t) \leq \frac{V\left(\tau_{i-1}\right)}{z\left(\tau_{i-1}\right)} z(t) .
$$

By denoting $\delta_{0}=\frac{c_{1} z\left(t_{0}\right) \theta}{c_{2}\left(1+\max _{j \in N}\left\{z\left(\tau_{j}\right)\right\}\right) \prod_{j=1}^{\infty}\left(1 \vee \omega_{j}\right)}$, it can be proved that when $\left\|x_{0}\right\|^{2}<\delta_{0}$,

$$
E\left(\left\|x\left(\tau_{i-1}\right)\right\|^{2}\right)<\theta
$$

Holds for all $i=1,2, \ldots$, and

$$
V(t, x(t)) \leq V\left(t_{0}, x\left(t_{0}\right)\right) \frac{z(t)}{z\left(t_{0}\right)} \prod_{j=0}^{i-1} \omega_{j}
$$

holds for all $t \in\left[\tau_{i-1}, \tau_{i}\right)$ by mathematical induction. From (12), it is obviously true for $i=1$. Assume that (14) is true for any $i \geq 1$, then for all $t \in\left[\tau_{i-1}, \tau_{i}\right)$ ), it is true that

$$
E\left(\left\|x\left(\tau_{i-1}\right)\right\|^{2}\right) \leq \frac{\theta z\left(\tau_{i-1}\right)}{\left(1+\max _{j \in N}\left\{z\left(\tau_{j}\right)\right\}\right) \prod_{j=i}^{\infty}\left(1 \vee \omega_{j}\right)}<\theta
$$

and

$$
V(t, x(t)) \leq V\left(t_{0}, x\left(t_{0}\right)\right) \frac{z(t)}{z\left(t_{0}\right)} \prod_{j=0}^{i-1} \omega_{j}
$$

From assumption (iii), we have that

$$
E\left(\left\|x\left(\tau_{i}\right)\right\|^{2}\right) \leq \frac{\theta z\left(\tau_{i}\right)}{\left(1+\max _{j \in N}\left\{z\left(\tau_{j}\right)\right\}\right) \prod_{j=i+1}^{\infty}\left(1 \vee \omega_{j}\right)}<\theta .
$$

Then by (ii),

$$
V(t) \leq V\left(\tau_{i}\right) \frac{z(t)}{z\left(\tau_{i}\right)} \leq \omega_{i} V\left(\tau_{i}^{-}\right) \frac{z(t)}{z\left(\tau_{i}\right)} \leq V\left(t_{0}\right) \frac{z(t)}{z\left(t_{0}\right)} \prod_{j=1}^{i} \omega_{j}
$$

for $z\left(\tau_{i}\right)=z\left(\tau_{i}^{-}\right)$. Then by mathematical induction (14) is true for $i=1,2, \ldots$. Combining $z(t) \leq 1$, (iv) (vi) and the above results,

$$
\begin{aligned}
V(t) \leq \frac{V\left(t_{0}\right)}{z\left(t_{0}\right)} \rho^{i-1} & =\frac{V\left(t_{0}\right)}{\rho z\left(t_{0}\right)} \exp (-i|\ln \rho|) \\
& \leq \frac{V\left(t_{0}\right)}{\rho^{t_{0}+1} z\left(t_{0}\right)} \exp \left(-\tau_{i}|\ln \rho|\right) \\
& \leq \frac{V\left(t_{0}\right)}{\rho^{t_{0}+1} z\left(t_{0}\right)} \exp (-t|\ln \rho|)
\end{aligned}
$$


since $\tau_{i-1} \leq t \leq \tau_{i} \leq t_{0}+i$.

Therefore $E\left(\|x(t)\|^{2}\right) \leq \frac{c_{1} E\left(\left\|x_{0}\right\|^{2}\right)}{c_{2} \rho^{t_{0}+1}} \exp (-t|\ln \rho|)$ holds. The proof is complete.

Remark 1. Theorem 3.3 is not a simple corollary of Theorem 3.1, since the conditions (ii) and (v) in Theorem 3.3 is weaker than that in Theorem 3.1.

Remark 2. Theorem 3.3 shows that $\int_{0}^{\infty} k(s) \exp (\gamma s) \mathrm{d} s<\infty$ is not necessary condition for exponential asymptotical stability, which can also be found in Theorem 3.5.

\subsection{Mean square non-exponential asymptotic stability}

To show that the solution of (1) is mean square non-exponentially asymptotically stable, we have to prove that $\lim _{t \rightarrow \infty} E\left(\|x(t)\|^{2}\right)=0$ and $\lim _{t \rightarrow \infty} \frac{\log \left(E\left(\|x(t)\|^{2}\right)\right)}{t}=0$. Now we prove the solution convergent to zero firstly.

Theorem 3.4. If there exist positive numbers $c_{1}, c_{2}$ and $V \in C^{1,2}\left(R^{+} \times R^{n}, R^{+}\right)$ satisfying

(i) $c_{1}\|x\|^{p} \leq V(t, x) \leq c_{2}\|\mathrm{x}\|^{p}$;

(ii) there exist two continuous and integrable functions $k, h: R^{+} \rightarrow R^{+}$such that for any $i=1,2, \ldots .$.

$$
L V(t, x(t)) \leq-a V(t, x(t))+\int_{\tau_{i}}^{t} k(t-s) V(s, x(s)) \mathrm{d} s+h(t), t \in\left(\tau_{i-1}, \tau_{i}\right)
$$

holds for some constant $a>0$;

(iii) there exist constants $\omega_{i}$ such that for any $i=1,2, \ldots . .$. , we have

$$
E V\left(\tau_{i}, x\left(\tau_{i}\right)\right) \leq \omega_{i} E V\left(\tau_{i}, x\left(\tau_{i}^{-}\right)\right) ;
$$

(iv) there exists $0<\rho<1$ such that $\max _{j \in N \backslash\{0\}}\left\{\omega_{j}\right\} \leq \rho$;

(v) $\int_{0}^{\infty} k(s) \mathrm{d} s \leq a$.

Then zero solution of (1) is mean square asymptotically stable.

Proof. From (11), by Lemma 2.2 and by Lemma 2.3,

$$
V(t) \leq \frac{V\left(t_{0}\right)}{z\left(t_{0}\right)} \rho^{i-1}+\int_{\tau_{i-1}}^{t} z(t-s) h(s) d s+\sum_{l=1}^{i-1} \rho^{i-1-j} \int_{\tau_{l-1}}^{\tau_{l}} z\left(\tau_{l}-s\right) h(s) \mathrm{d} s
$$

for $t \in\left[\tau_{i-1}, \tau_{i}\right)$. Noticing $0<\rho<1$, for any $\varepsilon>0$, there is $k_{0}>0$ such that $\rho^{k}<\frac{c_{1} \varepsilon}{4 M_{0}}$ where $M_{0}=\frac{V\left(t_{0}\right)}{z\left(t_{0}\right)}+\frac{1}{1-\rho}+\int_{0}^{\infty} h(s) \mathrm{d} s>1$. For $h(t)$ is integrable, for any $\varepsilon$ defined above, there is $\bar{k}>0$ such that $\int_{\tau_{k-1}}^{\infty} h(s) \mathrm{d} s<\frac{c_{1} \varepsilon}{4 M_{0}}$ for $k>\bar{k}$. It follows that 


$$
\begin{aligned}
\sum_{l=1}^{i-1} \rho^{i-1-j} \int_{\tau_{l-1}}^{\tau_{l}} h(s) \mathrm{d} s & =\sum_{l=1}^{\bar{k}} \rho^{i-1-j} \int_{\tau_{l-1}}^{\tau_{l}} h(s) \mathrm{d} s+\sum_{l=\bar{k}+1}^{i-1} \rho^{i-1-j} \int_{\tau_{l-1}}^{\tau_{l}} h(s) \mathrm{d} s \\
& \leq \frac{\varepsilon c_{1}}{4 M_{0}} \sum_{j=0}^{\infty} \rho^{j}+\frac{\varepsilon c_{1}}{4 M_{0}} \int_{t_{0}}^{\infty} h(s) \mathrm{d} s \\
& \leq \frac{\varepsilon c_{1}}{4 M_{0}}\left(\frac{1}{1-\rho}+\int_{t_{0}}^{\infty} h(s) \mathrm{d} s\right)
\end{aligned}
$$

for $i>\bar{k}+k_{0}+1$. By choosing $\delta=\frac{1}{c_{2}}$, it follows (15) directly that for any $\varepsilon>0$, we have $E\left(\|x(t)\|^{2}\right)<\varepsilon$ for $t>\tau_{\bar{k}+k_{0}+2}$ when $E\left(\left\|x_{0}\right\|^{2}\right)<\delta$. The proof is complete.

Theorem 3.5. If there exist positive numbers $c_{1}, c_{2}$ and $V \in C^{1,2}\left(R^{+} \times R^{n}, R^{+}\right)$satisfying (i)-(v) in theorem 3.3 and

(H1) there exist continuous and integrable function $\bar{h}: R^{+} \rightarrow R^{+}$satisfying $\bar{h}(t) \leq h(t)$ and constant àsatisfying $\bar{a} \geq$ asuch that

$$
L V(t, x(t)) \geq-a V(t, x(t))+\bar{h}(t), t \in\left(\tau_{i-1}, \tau_{i}\right)
$$

for any $i=1,2, \ldots$;

(H2) there exist constants $0 \leq \bar{\omega}_{i} \leq \omega_{i}$ such that for any $i=1,2, \ldots$, we have

$$
E V\left(\tau_{i}, x\left(\tau_{i}\right)\right) \geq \bar{\omega}_{i} E V\left(\tau_{i}, x\left(\tau_{i}^{-}\right)\right)
$$

(H3) $\bar{h}(t) \in C^{1}[0,+\infty)$ satisfies $\bar{h}(t)>0$ and $\lim _{t \rightarrow \infty} \frac{\bar{h}^{\prime}(t)}{\bar{h}(t)}=0$;

(H4) there is constant $1>d>0$ such that $\min _{j \in N \backslash\{0\}}\left\{\bar{\omega}_{j}\right\} \geq d$;

(H5) $\log \left(\tau_{i}-t_{0}\right) \geq i$ for all $i=1,2, \ldots$.

Then zero solution of (1) is mean square non-exponentially asymptotically stable.

Proof. By use of Theorem 3.3, we obtain

$$
\lim _{t \rightarrow \infty} E\left(\|x(t)\|^{2}\right)=0 .
$$

From $(H 1)$, we have that

$$
V(t) \geq V\left(\tau_{i-1}\right) \exp (-\bar{a} t)+\int_{\tau_{i-1}}^{t} \exp (-\bar{a}(t-s)) \bar{h}(s) \mathrm{d} s
$$

for all $t \in\left[\tau_{i-1}, \tau_{i}\right)$. Consequently, we get

$$
\begin{aligned}
V(t) \geq \exp \left(-\bar{a}\left(t-\tau_{0}\right)\right) & \prod_{j=1}^{i-1} \omega_{j}+\int_{\tau_{i-1}}^{t} \exp (-\bar{a}(t-s)) \bar{h}(s) \mathrm{d} s \\
& +\sum_{j=1}^{i-1} \prod_{l=j}^{i} \omega_{l} \int_{\tau_{j-1}}^{\tau_{j}} \exp (-\bar{a}(t-s)) \bar{h}(s) \mathrm{d} s
\end{aligned}
$$

for all $t \in\left[\tau_{i-1}, \tau_{i}\right)$ for $i=1,2, \ldots$ by mathematical induction from (H1)-(H2). From assumption 


$$
V(t) \geq \frac{d^{i-1} \int_{\tau_{0}}^{t} \exp (\bar{a} s) \bar{h}(s) \mathrm{d} s}{\exp (\bar{a} t)} .
$$

Since $\bar{h}(t) \in C^{1}[0,+\infty)$ satisfies $\bar{h}(t)>0$ and $\lim _{t \rightarrow \infty} \frac{\bar{h}^{\prime}(t)}{\bar{h}(t)}=0$, then

$\lim _{t \rightarrow \infty} \inf \exp (\bar{a} t) \bar{h}(t)=\infty$.

By L'Hospital rule

$$
\begin{aligned}
\lim _{t \rightarrow \infty} \inf \frac{1}{t} \log \frac{\int_{\tau_{0}}^{t} \exp (\bar{a} s) \bar{h}(s) \mathrm{d} s}{\exp (\bar{a} t)} & \geq-\bar{a}+\lim _{t \rightarrow \infty} \inf \frac{\log \int_{\tau_{0}}^{t} \exp (\bar{a} s) \bar{h}(s) \mathrm{d} s}{t} \\
& \geq-\bar{a}+\lim _{t \rightarrow \infty} \inf \frac{\exp (\bar{a} t) \bar{h}(t)}{\int_{\tau_{0}}^{t} \exp (\bar{a} s) \bar{h}(s) \mathrm{d} s} \\
& \geq-\bar{a}+\lim _{t \rightarrow \infty} \inf \frac{\bar{a} \exp (\bar{a} t) \bar{h})(t)+\exp (\bar{a} t) \bar{h}^{\prime}(t)}{\exp (\bar{a} t) \bar{h}(t)} \\
& =0 .
\end{aligned}
$$

From (H5) and $d<1$,

$$
\begin{aligned}
\lim _{t \rightarrow \infty} \inf \frac{\log \left(d^{i-1}\right)}{t} & \geq \lim _{t \rightarrow \infty} \inf \frac{i \log (d)-\log (d)}{t} \\
& \geq \lim _{t \rightarrow \infty} \inf \frac{\log \left(t-t_{0}\right) \log (d)-\log (d)}{t} \\
& =0 .
\end{aligned}
$$

Thus, combine (16) and (17) to show

$$
\lim _{t \rightarrow \infty} \inf \frac{\log E\left(\|x(t)\|^{2}\right)}{t} \geq \lim _{t \rightarrow \infty} \inf \frac{\log V(t)-\log c_{2}}{t} \geq 0 .
$$

Since $\lim _{t \rightarrow \infty} E\left(\|x(t)\|^{2}\right)=0$ holds under assumptions (i)-(v) in Theorem 3.3, we get

$$
\lim _{t \rightarrow \infty} \sup \frac{\log E\left(\|x(t)\|^{2}\right)}{t} \leq 0
$$

Therefore

$$
\lim _{t \rightarrow \infty} \frac{\log E\left(\|x(t)\|^{2}\right)}{t}=0 .
$$

The proof is complete.

Remark 3. Assumption (H5) in Theorem 3.5 can be replaced by $\lim _{i \rightarrow \infty} \frac{i}{\tau_{i}}=0$.

\section{Example}

Example 1. Consider a nonlinear impulsive stochastic Volterra equation of the form

$$
\left\{\begin{array}{l}
\mathrm{d} x_{1}=\left(-x_{1}+x_{2} \int_{\tau_{k}}^{t} x_{2} x_{1} \exp (t-s) \mathrm{d} s\right)+\sqrt{\int_{\tau_{k}}^{t}\left(x_{1}^{2}+x_{2}^{2}\right) \exp (-3(t-s)) \mathrm{d}} d B_{1}(t) \\
\mathrm{d} x_{2}=\left(-5 x_{2}-x_{1} \int_{\tau_{k}}^{t} x_{1} x_{2} \exp (t-s) \mathrm{d} s\right)+\exp (-\sqrt{t}) d B_{2}(t)
\end{array}\right.
$$


for $t \in\left(\tau_{k}, \tau_{k}+1\right)$ with $\left\{\begin{array}{l}x_{1}(0)=1 \\ x_{2}(0)=1\end{array}\right.$, where $\tau_{k}=2^{k}$ and the impulse is defined as

$$
\begin{aligned}
& x_{1}\left(\tau_{k}\right)=x_{1}\left(\tau_{k}\right) \lambda_{1}(k) \\
& x_{2}\left(\tau_{k}\right)=x_{2}\left(\tau_{k}\right) \lambda_{2}(k)
\end{aligned}
$$

for all $k \in N . \lambda_{1}(k)$ and $\lambda_{2}(k)$ are random variables on $\left[\frac{1}{6}, \frac{3}{4}\right]$. Then the zero solution of (18) and (19) is mean square non-exponentially asymptotically stable.

Proof. By putting $V(t, x(t))=\|x(t)\|^{2}=x_{1}^{2}+x_{2}^{2}$, we have that

$$
\begin{aligned}
L V(t, x(t))=2 & x_{1}\left(-x_{1}+x_{2} \int_{\tau_{k}}^{t} x_{2} x_{1} \exp (t-s) \mathrm{d} s\right) \\
& +2 x_{2}\left(-5 x_{2}+x_{1} \int_{\tau_{k}}^{t} x_{1} x_{2} \exp (t-s) \mathrm{d} s\right) \\
& +\int_{\tau_{k}}^{t}\left(x_{1}^{2}+x_{2}^{2}\right) \exp (-3(t-s)) \mathrm{d} s+\exp (-2 \sqrt{t}) \\
=- & \left(2 x_{1}^{2}+10 x_{2}^{2}\right)+\int_{\tau_{k}}^{t}\left(x_{1}^{2}+x_{2}^{2}\right) \exp (-3(t-s)) \mathrm{d} s+\exp (-2 \sqrt{t})
\end{aligned}
$$

It follows that

$$
L V(t, x(t)) \leq-2 V(t, x(t))+\int_{\tau_{k}}^{t}\left(x_{1}^{2}+x_{2}^{2}\right) \exp (-3(t-s)) \mathrm{d} s+\exp (-2 \sqrt{t}) .
$$

Since

$$
E V\left(\tau_{k}, x\left(\tau_{k}\right)\right)=E\left(x_{1}^{2}\left(\tau_{k}\right)+x_{2}^{2}\left(\tau_{k}\right)\right)=E\left(\lambda_{1}^{2}(k) x_{1}^{2}\left(\tau_{k}^{-}\right)+\lambda_{2}^{2}(k) x_{2}^{2}\left(\tau_{k}^{-}\right)\right),
$$

we have

$$
\frac{1}{36} \leq \bar{\omega}_{j} \leq \omega_{j} \leq \frac{9}{16}
$$

for all $j>0$. In addition,

$$
\int_{0}^{\infty} k(s) \exp (2 s) \mathrm{d} s=\int_{0}^{\infty} \exp (-3 s) \exp (2 s) \mathrm{d} s=1<2
$$

and

$$
\int_{0}^{\infty} \exp (-2 \sqrt{s}) \mathrm{d} s=\frac{1}{2}<\infty
$$

hold. By Theorem 3.4, it is true that

$$
\lim _{t \rightarrow \infty} E\left(\|x(t)\|^{2}\right)=0 .
$$


Next, we prove

$$
\lim _{t \rightarrow \infty} \frac{\log E\left(\|x(t)\|^{2}\right)}{t}=0 .
$$

From (20),

$$
\begin{aligned}
& \qquad V(t, x(t)) \geq-10 V(t, x(t))+\exp (-2 \sqrt{t}) \\
& \text { Since } \lim _{t \rightarrow \infty} \frac{(\exp (-2 \sqrt{t}))^{\prime}}{\exp (-2 \sqrt{t})}=\lim _{t \rightarrow \infty} \frac{1}{-\sqrt{t}}=0 \text { and } \log \tau_{k}=k \text {, we finish the proof by The- }
\end{aligned}
$$

orem 3.5.

\section{Acknowledgements}

The authors sincerely thank the anonymous reviewer for his careful reading, constructive comments and fruitful suggestions to improve the quality of the manuscript. This article is partially supported by NSFC (No. 11001173).

\section{Author details}

${ }^{1}$ Department of mathematics, Shanghai Jiaotong University, Shanghai, 200240, China ${ }^{2}$ College of Science, University of Shanghai for Science and Technology, Shanghai, 200093, China

\section{Authors' contributions}

DZ and DH conceived of the study. DZ carried out most of the analysis and drafted the manuscript. DH revised and commented on the draft. All authors read and approved the final manuscript.

\section{Competing interests}

The authors declare that they have no competing interests.

Received: 11 October 2010 Accepted: 17 June 2011 Published: 17 June 2011

\section{References}

1. Taniguchi, T: The exponential stability for stochastic delay partial differential equations. J Math Anal Appl. 331, 191-205 (2007). doi:10.1016/j.jmaa.2006.08.055

2. Wan, L, Duan, J: Exponential stability of non-autonomous stochastic partial differential equations with finite memory. Stat Probab Lett. 78, 490-498 (2008). doi:10.1016/j.spl.2007.08.003

3. Luo, J: Fixed points and exponential stability of mild solutions of stochastic partial differential equations with delays. J Math Anal Appl. 342, 753-760 (2008). doi:10.1016/j.jmaa.2007.11.019

4. Sakthivel, R, Luo, J: Asymptotic stability of nonlinear impulsive stochastic differential equations. Stat Probab Lett. 79, 1219-1223 (2009). doi:10.1016/j.spl.2009.01.011

5. Sakthivel, R, Luo, J: Asymptotic stability of impulsive stochastic partial differential equations with infinite delays. J Math Anal Appl. 356, 1-6 (2009). doi:10.1016/j.jmaa.2009.02.002

6. Brauer, F: Asymptotic stability of a class of integro-differential equations. J Diff Equ. 28, 180-188 (1978). doi:10.1016/ 0022-0396(78)90065-7

7. Burton, TA, Mahfoud, WE: Stability criterion for Volterra equations. Trans Am Math Soc. 279, 143-174 (1983). doi:10.1090/S0002-9947-1983-0704607-8

8. Kordonis, IGE, Philos, ChG: The behavior of solutions of linear integro-differential equations with unbounded delay. Comput Math Appl. 38, 45-50 (1999). doi:10.1016/S0898-1221(99)00181-9

9. Murakami, S: Exponential asymptotic stability for scalar linear Volterra equations. Diff Integral Equ. 4(2):519-525 (1991)

10. Appleby, JAD, Reynolds, DW: On necessary and sufficient conditions for exponential stability in linear Volterra integrodifferential equations. J Integral Equ Appl. 16(3):221-240 (2004). doi:10.1216/jiea/1181075283

11. Appleby, JAD, Reynolds, DW: Subexponential solutions of linear integro-differential equations and transient renewal equations. Proc R Soc Edinb. 132A, 521-543 (2002)

12. Appleby, JAD, Reynolds, DW: On the non-exponential convergence of asymptotically stable solutions of linear scalar Volterra integro-differential equations. J Integral Equ Appl. 14(2):109-118 (2002). doi:10.1216/jiea/1031328362

13. Mao, X, Riedle, M: Mean square stability of stochastic Volterra integro-differential equations. Syst Control Lett. 55, 459-465 (2006). doi:10.1016/j.sysconle.2005.09.009

14. Appleby, JAD, Reynolds, DW: Non-exponential stability of scalar stochastic Volterra equations. Statist Probab Lett. 62(4):335-343 (2003). doi:10.1016/S0167-7152(03)00035-X

15. Appleby, JAD, Riedle, M: Almost sure asymptotic stability of stochastic Volterra integro-differential equations with fading perturbations. Stoch Anal Appl. 24(4):813-826 (2006). doi:10.1080/07362990600753536

16. Appleby, JAD: Subexponential solutions of scalar linear Ito-Volterra equations with damped stochastic perturbations. Funct Differ Equ. 11(1-2):5-10 (2004)

17. Appleby, JAD: Almost sure subexponential decay rates of scalar Ito-Volterra equations. Electron J Qual Theory Differ Equ., Proc 7th Coll. QTDE, Paper no 1. 1-32 (2004)

18. Appleby, JAD, Freeman, A: Exponential asymptotic stability of linear Ito-Volterra equations with damped stochastic perturbations. Electron J Probab. 8(22):22 (2003) 
19. Appleby, JAD, Reynolds, DW: Decay rates of solutions of linear stochastic Volterra equations. Electron J Probab. 13(30):922-943 (2008)

20. Becker, LC: Function bounds for solutions of Volterra equations and exponential asymptotic stability. Nonlinear Anal. 67, 382-397 (2007). doi:10.1016/.na.2006.05.016

doi:10.1186/1029-242X-2011-9

Cite this article as: Zhao and Han: Mean square exponential and non-exponential asymptotic stability of

impulsive stochastic Volterra equations. Journal of Inequalities and Applications 2011 2011:9.

Submit your manuscript to a SpringerOpen ${ }^{\odot}$ journal and benefit from:

- Convenient online submission

- Rigorous peer review

- Immediate publication on acceptance

- Open access: articles freely available online

- High visibility within the field

- Retaining the copyright to your article

Submit your next manuscript at $\gg$ springeropen.com 